Proc. Indian Acad. Sci. (Earth Planet. Sci.), Vol. 97, No. 2, December 1988, pp. 137-147.

(C) Printed in India.

\title{
Spectra of different fluxes over the arid and semiarid zones of northwest India during MONEX 1979
}

\author{
U S SINGH, DAYA SHANKER and R K SINGH \\ Department of Geophysics, Banaras Hindu University, Varanasi 221005 , India \\ MS received 14 March 1988
}

\begin{abstract}
Fluxes of heat, momentum and the kinetic energy over the and and semiarid regions of Indian Peninsula have been computed. Spectral analysis of these fluxes at $50 \mathrm{mb}$ interval were performed at each level in the vertical up to $300 \mathrm{mb}$ surface. The eddy latent beat transport is governed essentially by short- and long-range periodicities. A S-day periodicity is generally present in the vertical mean eddy fluxes. Long-range periodic oscillations are more pronounced in the extreme north of the Indian subcontinent. Dominance of medium-range periodicity is observed in all the fluxes except latent heat.
\end{abstract}

Keywords. Atmospheric flux; periodicity; MONEX; vertical distribution; oscillations.

\section{Introduction}

The study of different fluxes helps in better understanding of excess and deficit in the budget analysis. Spectral analysis has been widely used to understand the dominant periodicities in meteorological parameters to study the cause of the oscillations. Several investigators (Sankar Rao 1962; Sankar Rao and Ramanadham 1963; Saha 1970; Saha and Bavadekar 1973, 1977; Rao and Ramanamurty 1977; Ghosh et al 1978; Bavadekar and Mooley 1978; Rao 1981) attempted to study the fluxes over the Indian region. Singh and Singh (1987) carried out the study of heat and momentum transport over the east and west coasts of India during MONEX 79. The power spectral analysis of useful meteorological variables has been attempted by many workers. Madden and Julian (1972) showed the evidence of a global 5-day pressure wave. Gupta (1972) and Gupta and Singh (1977) observed the 5-day pressure oscillation over the Siberian anticyclone and the 4-5-day pressure oscillation over the Mediterranean regions respectively.

The present study was carried out to throw some light on the significant periodicities in sensible heat fluxes, latent heat fluxes and momentum and kinetic energy fluxes over the arid and semiarid zones of northwest India during the MONEX 79 summer monsoon months May to August at each level in the vertical up to $300 \mathrm{mb}$ surface.

\section{Data and method of analysis}

The daily aerological data obtained from the MONEX 79 set of data at Ahmedabad, Jodhpur, Gwalior, New Delhi and Patiala have been used for computation of sensible heat transport, latent heat transport, momentum transport and flux of kinetic energy. These vertically integrated fluxes, as well as fluxes at each level up to $300 \mathrm{mb}$ surface, 
were subjected to spectral analysis in time domain following Blackman and Tukey (1958).

The heat, momentum and kinetic energy fluxes at any station may be expressed as

$$
\begin{aligned}
& Q=\frac{C_{P}}{g} \int_{0}^{P_{0}} \bar{V} \bar{T} \mathrm{~d} P+\frac{L}{g} \int_{0}^{P_{0}} \bar{V} \bar{X} \mathrm{~d} P+\frac{C_{P}}{g} \int_{0}^{P_{0}} \overline{V^{\prime} T^{\prime}} \mathrm{d} P+\frac{L}{g} \int_{0}^{P_{0}} \overline{V^{\prime} X^{\prime}} \mathrm{d} P \\
& M=\frac{1}{g} \int_{0}^{P_{0}} \bar{U} \bar{V} \mathrm{~d} P+\frac{1}{g} \int_{0}^{P_{0}} \overline{U^{\prime} V^{\prime}} \mathrm{d} P \\
& K=\bar{K}+K^{\prime} ; \quad \bar{K}=\frac{1}{2}\left(\bar{U}^{2}+\bar{V}^{2}\right) ; \quad K^{\prime}=\frac{1}{2}\left(U^{\prime 2}+V^{\prime 2}\right) \\
& F_{k}=\int_{0}^{P_{0}} \bar{V} \bar{K} \mathrm{~d} P+\int_{0}^{P_{0}} V^{\prime} K^{\prime} \mathrm{d} P,
\end{aligned}
$$

where $Q$ is the time-averaged northward flux of sensible and latent heat, $M$ the momentum flux per unit time, $X$ the humidity mixing ratio, $K$ the kinetic energy and $F_{k}$ the flux of kinetic energy per unit mass. The other parameters have their usual meaning. $\left({ }^{-}\right)$denotes the time average and ()$^{\prime}$ the deviation from the time average.

\section{Discussion of the results}

Of the several charts of power spectra, it has been considered adequate to present only the chart for vertical mean eddy flux in figures $1-5$ for the purpose of illustration.

Tables 1 and 2 show the existing periodicities over the arid and semiarid regions of India for eddy sensible heat transport and eddy momentum transport at Ahmedabad, Jodhpur, Gwalior, New Delhi and Patiala. The vertical distribution of periodicity in eddy kinetic energy and flux of eddy kinetic energies do not show any significant changes other than those periodicities in tables 1 and 2 . The computation of periodicity in eddy latent heat transport could not be performed from 700 to $300 \mathrm{mb}$ because of lack of suitable number of data in the time series. Hence they are not presented here. However, the results are summarized below.

The vertical mean eddy sensible heat transport (table 1) does not show any remarkable periodicity over Ahmedabad, Jodhpur and Gwalior. However, at New Delhi and Patiala the presence of a short-range (<5-day) or medium-range (5-10-day) periodicity in the vertical mean eddy sensible heat transport is observed. The vertical mean eddy latent heat transport shows the presence of medium-range periodicity over Ahmedabad, Jodhpur and Patiala and besides that a short-range periodicity is present at Gwalior and New Delhi. The observed periodicity in vertical mean eddy momentum transport varies from 4.7 to 5.6 days over these stations (table 2). Thus, a fluctuation at an average of about 5 days can be considered to be present in the vertical mean eddy momentum transport over these regions. The vertical mean eddy kinetic energy shows the presence of medium-range periodicity over Ahmedabad, Jodhpur and New Delhi whereas the vertical mean flux of kinetic energy shows periodicity of short range at Jodhpur and of medium range over New Delhi and Patiala.

Thus, the vertically integrated eddy sensible heat transport over Ahmedabad, Jodhpur and Gwalior during MONEX 79 seems to be due to eddies of different scales 


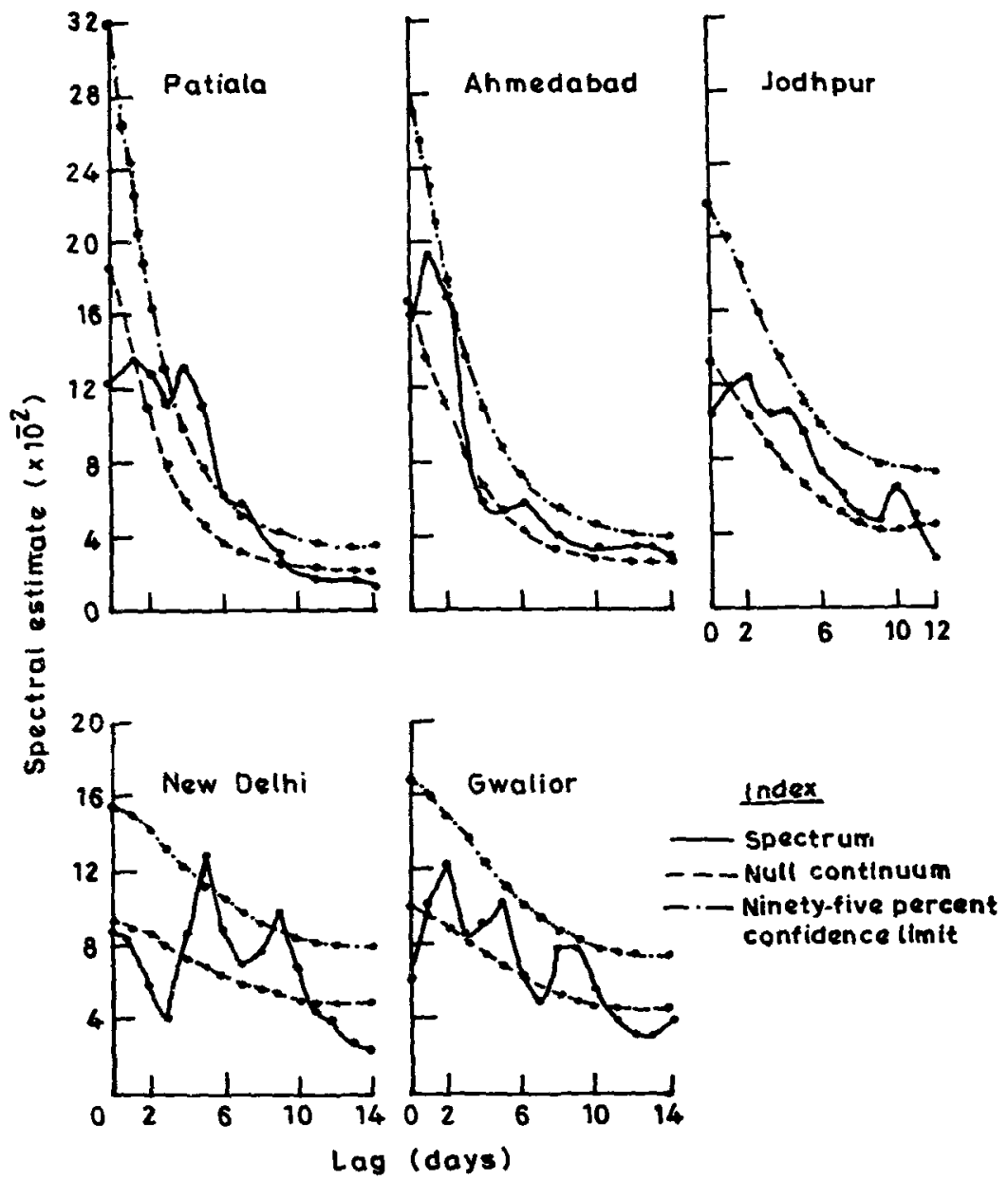

Figure 1. Spectra of vertical mean eddy sensible heat flux.

which do not show their periodic occurrence whereas the vertical mean of eddy momentum transport, eddy latent heat transport, eddy kinetic energy and eddy kinetic energy flux usually seem to be governed by atmospheric processes that have varying periodicity in short and medium ranges, more closely around 5 days. This 5-day periodicity in different meteorological parameters, studied by several investigators (Ananthakrishnan and Keshavamurty 1970; Bhalme and Parasnis 1975; Murakami 1976), is most prominent in northern India and is related to the frequency of monsoon lows travelling from the Bay of Bengal to northern India. Yanai et al (1968) found a westward-propagating wave with a 4-5-day period in certain regions of the tropics in the meridional component of the wind at lower tropospheric levels. Wallace and Chang (1969) found an easterly wave with a period of 4-5 days and also a 4-day period of planetary scale pressure fluctuation at the equator. Misra (1972) observed the westward-propagating planetary pressure wave of 4-5-day period in the tropics. Rao and Ramanamurty (1972) observed a 4-8-day periodicity in tropical wave disturbances over Singapore. 


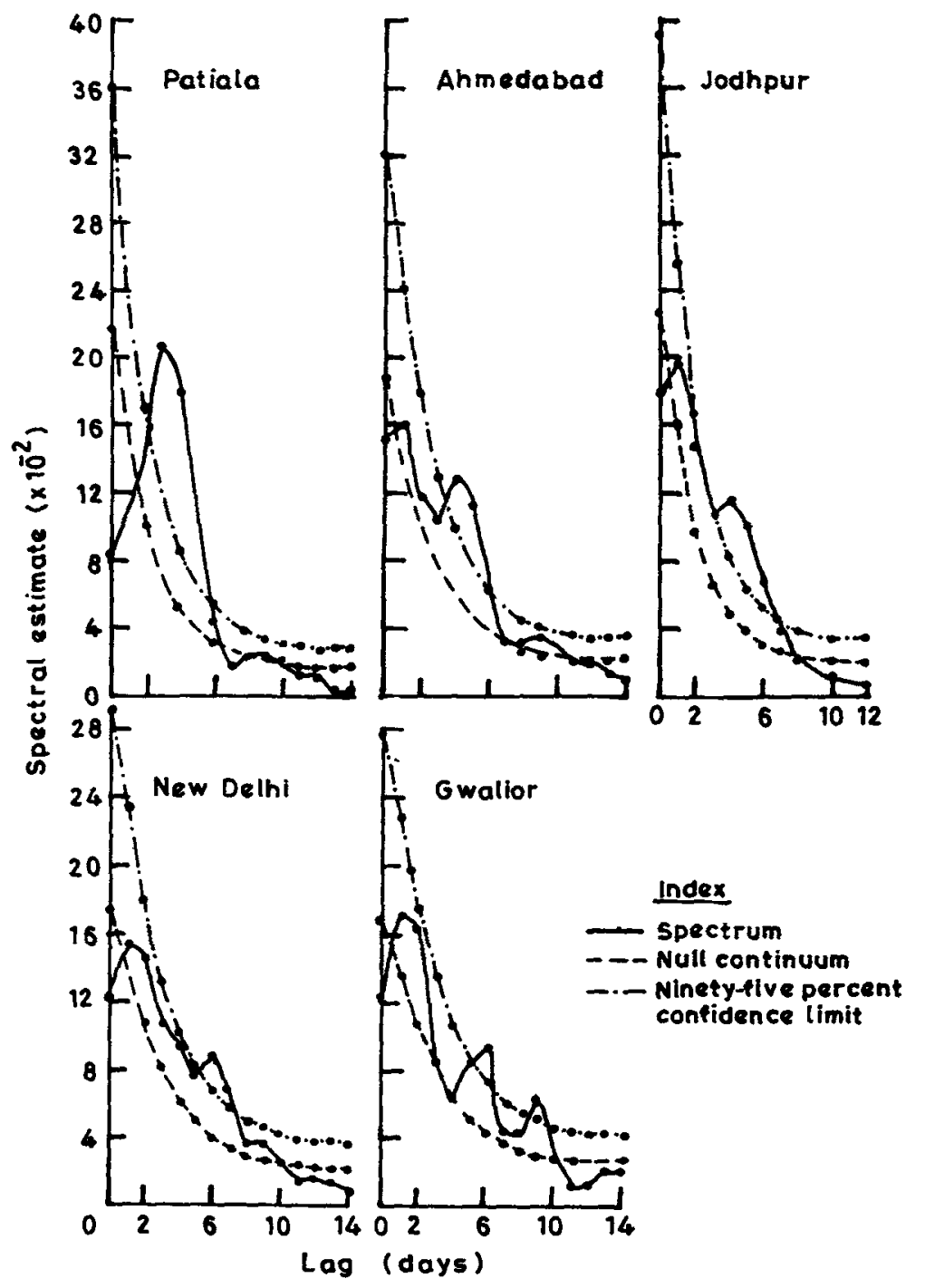

Figure 2. Spectra of vertical mean eddy latent heat flux.

We conclude that except in the eddy sensible heat transport over Ahmedabad, Jodhpur and Gwalior, a generally 3-7-day periodicity is present over the arid and semiarid zones of northwest India in vertical mean eddy latent heat transport, vertical mean eddy momentum transport and vertical mean eddy kinetic energy and vertical mean eddy flux of kinetic energy. Figure 6 and tables 1 and 2 show the vertical distribution of periodicities in eddy transport of heat, momentum and kinetic energy. In eddy latent heat transport short-, medium- and long-range ( $>10$-day) periodicity has been observed over the region. The eddy latent heat transport is dominated by short-and long-range periodic fluctuations of atomspheric processes in the layer below $800 \mathrm{mb}$ surface over Ahmedabad, Jodhpur, Gwalior and New Delhi. In the layer from 


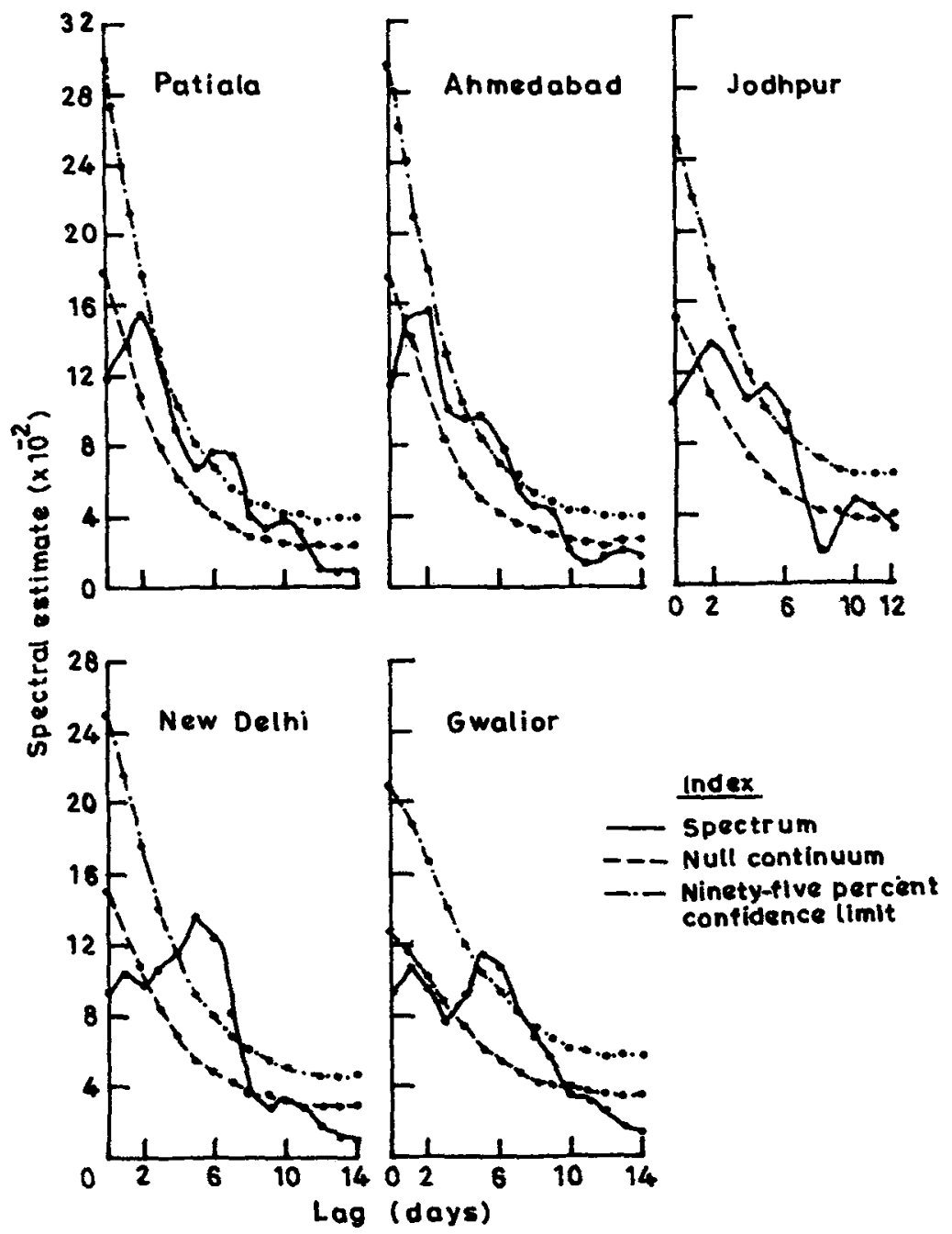

Figure 3. Spectra of vertical mean eddy momentum flux.

$800 \mathrm{mb}$ to $950 \mathrm{mb}$ surface over Gwalior and New Delhi, the eddy transport of latent heat is dominated by long-range periodicity.

The eddy sensible heat transport, eddy momentum transport, eddy kinetic energy and eddy flux of kinetic energy seem to be mainly controlled by short- and mediumrange fluctuations of atmospheric processes throughout the layer up to $300 \mathrm{mb}$ surface. The long-range periodicity is occasionally present below $700 \mathrm{mb}$ surface in these fluxes over Ahmedabad, Jodhpur, Gwalior and New Delhi. However, at Patiala we observe the presence of long-range periodicity at $900,850,700$ and $300 \mathrm{mb}$ in eddy momentum transport; at 850 and $900 \mathrm{mb}$ in eddy kinetic energy and at $900,700,400$ and $300 \mathrm{mb}$ in eddy flux of kinetic energy and at $750-650 \mathrm{mb}$ surface in eddy sensible heat transport. Thus, as we move towards the north over the arid and semiarid zones of northwest Indian subcontinent, the dominance of long-range periodicity is observed in the lower 


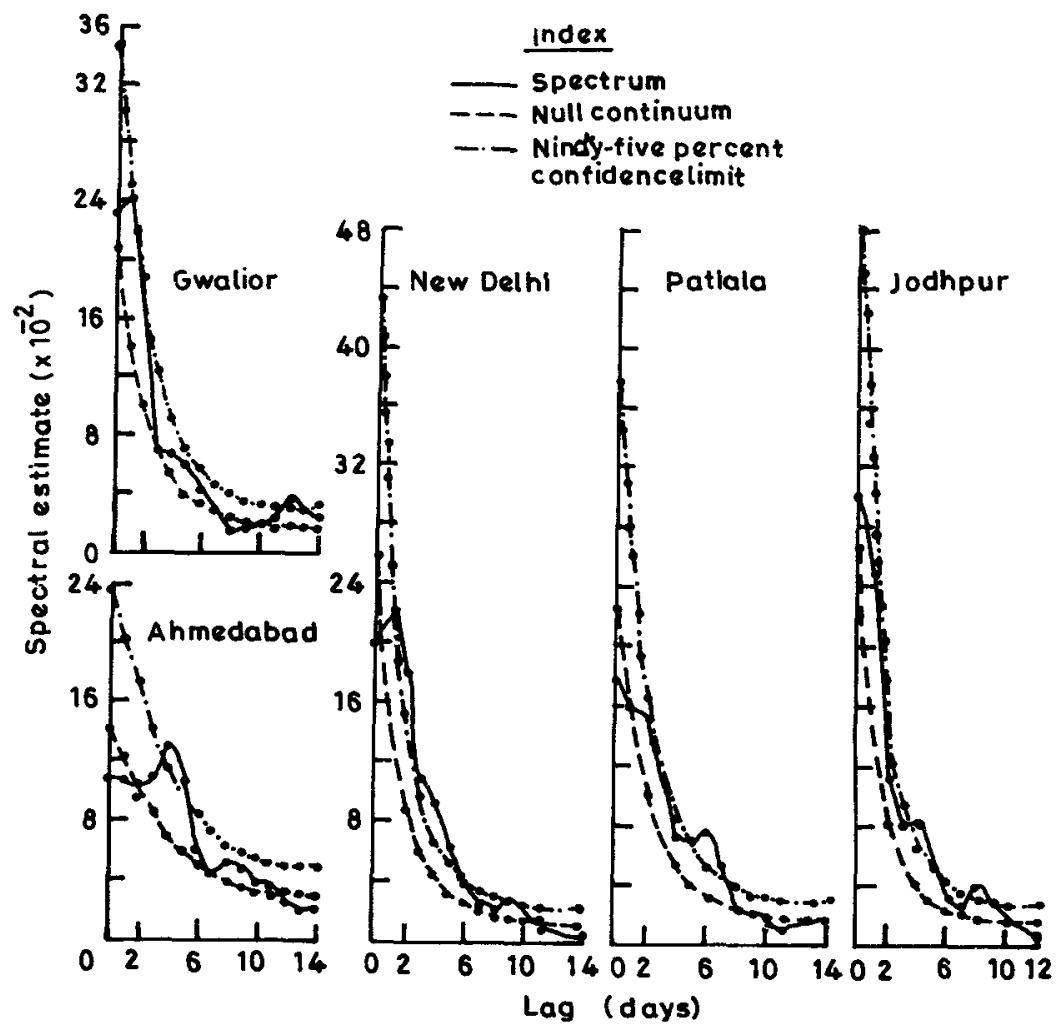

Figure 4. Spectra of vertical mean eddy kinetic energy.

troposphere around $700 \mathrm{mb}$ surface at Patiala, New Delhi and sometimes at Jodhpur in some of the fluxes, and at upper troposphere around $300-400 \mathrm{mb}$ surface.

At Ahmedabad and Gwalior, the long-range periodicity in eddy sensible heat transport is observed at about $300-400 \mathrm{mb}$ surface and at Patiala at about 650-750 $\mathrm{mb}$. This shows that with increasing latitude, the long-range periodicity, if present at upper surfaces (below $300 \mathrm{mb}$ surface), shifts to higher pressure surfaces (lower heights) over these regions. This is observed more clearly in eddy sensible heat transport and less clearly in other fluxes. These shifts seem to be due to the influence of western disturbances which passed through extreme northern Indian subcontinent during MONEX 79. As 1979 was an abnormal monsoon year, these fluxes may not be very representative of average monsoon conditions but still they throw much light on this area of research. Information on absolute and relative values of these fluxes can be useful for modelling studies. The noted periodicities in the range of 5-7 days and 12-15 days are in agreement with periodicities noted in the monsoon system by many earlier studies.

\section{Conclusions}

(i) A periodicity of around 5 days is substantiated over arid and semiarid zones of northwest Indian subcontinent in vertical mean eddy latent heat and momentum 


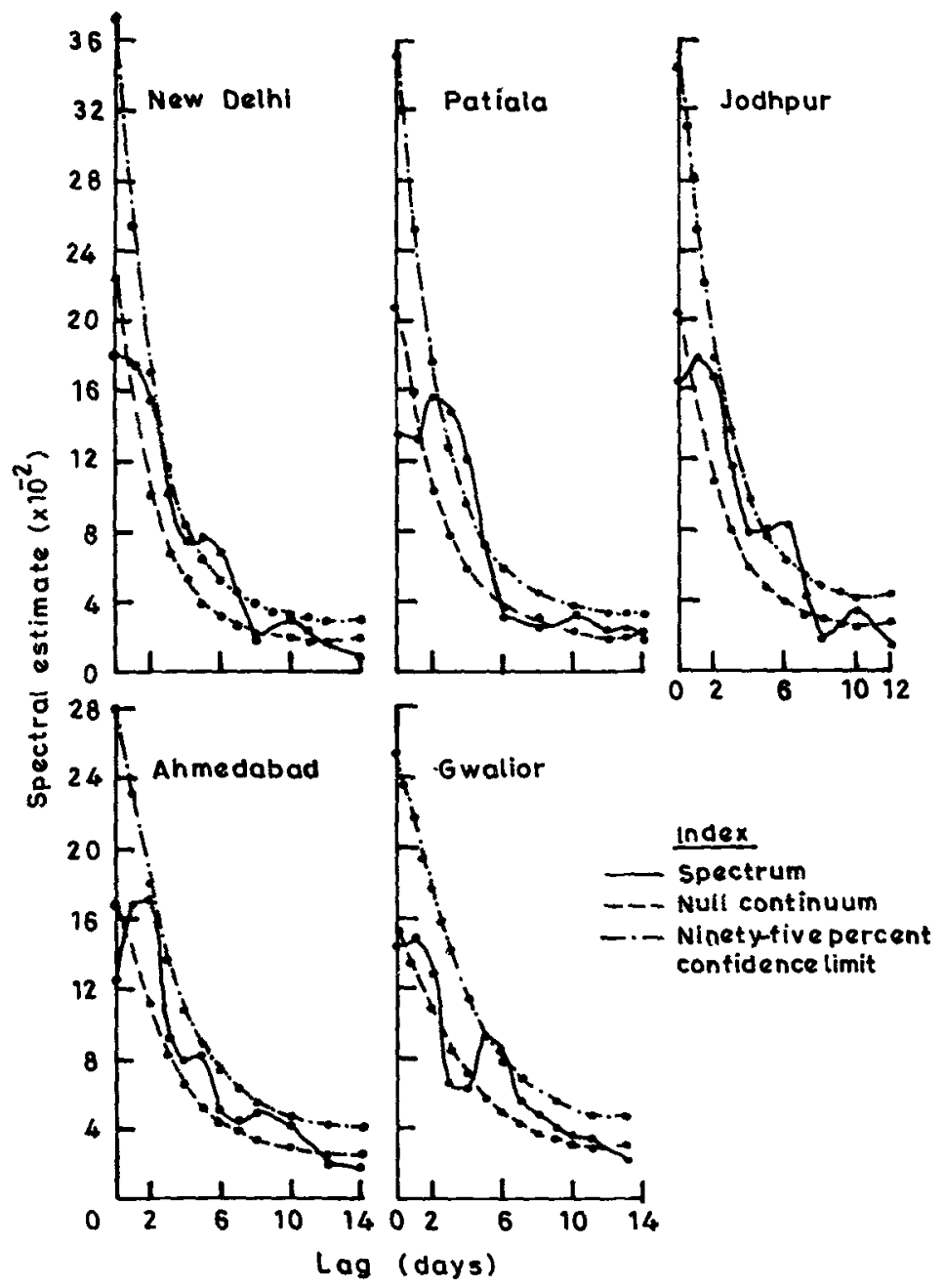

Figure 5. Spectra of vertical mean eddy kinetic energy flux.

transport. However, the vertical mean eddy sensible heat transport is not governed by any periodic oscillations over Ahmedabad, Jodhpur and Gwalior.

(ii) Medium-range periodicity usually dominates in eddy sensible heat, momentum, kinetic energy and flux of eddy kinetic energy in the lower troposphere. The eddy latent heat transport is essentially governed by short- and long-range periodicities over arid and semiarid zones of northwest India.

(iii) Long-range periodicity, if present at any surface, usually shifts to lower altitude pressure surface with increasing latitude over these regions of northwest India.

(iv) The dominance of medium-range periodicity in most of the fluxes in the lower troposphere as well as the shift of long-range periodicity to lower altitude with increasing latitude over northwest India seem to be associated with drought monsoon year. 


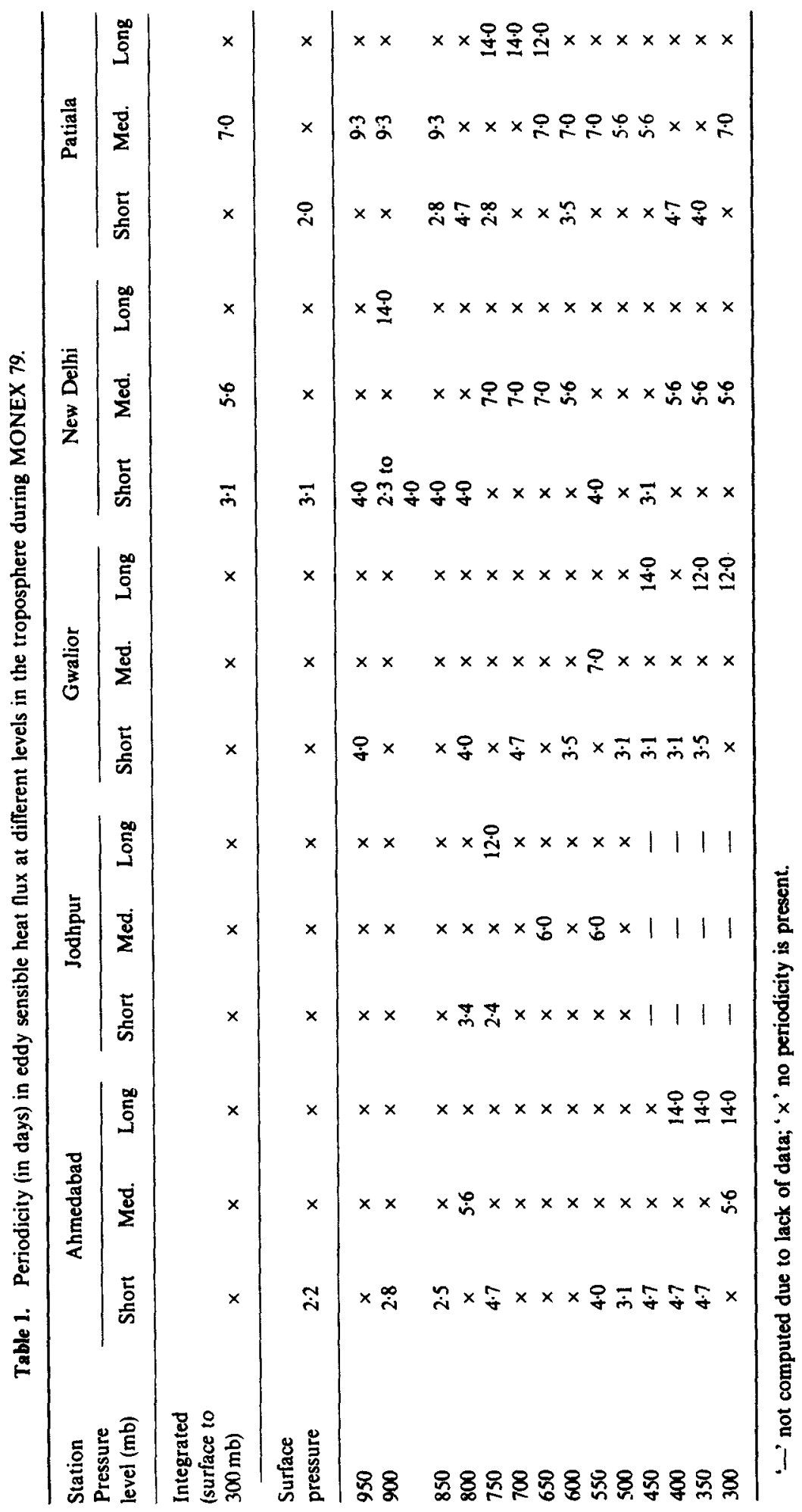




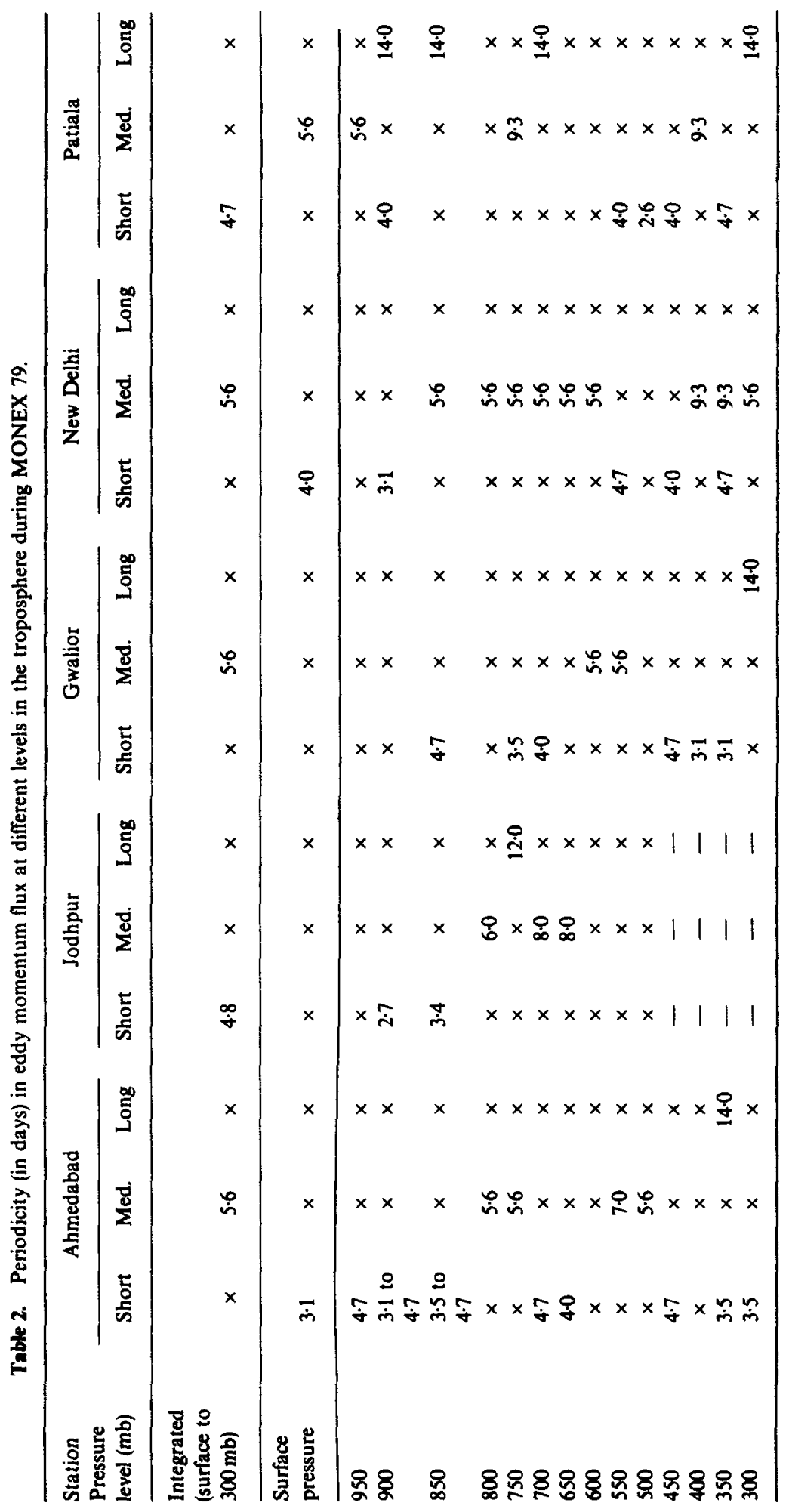




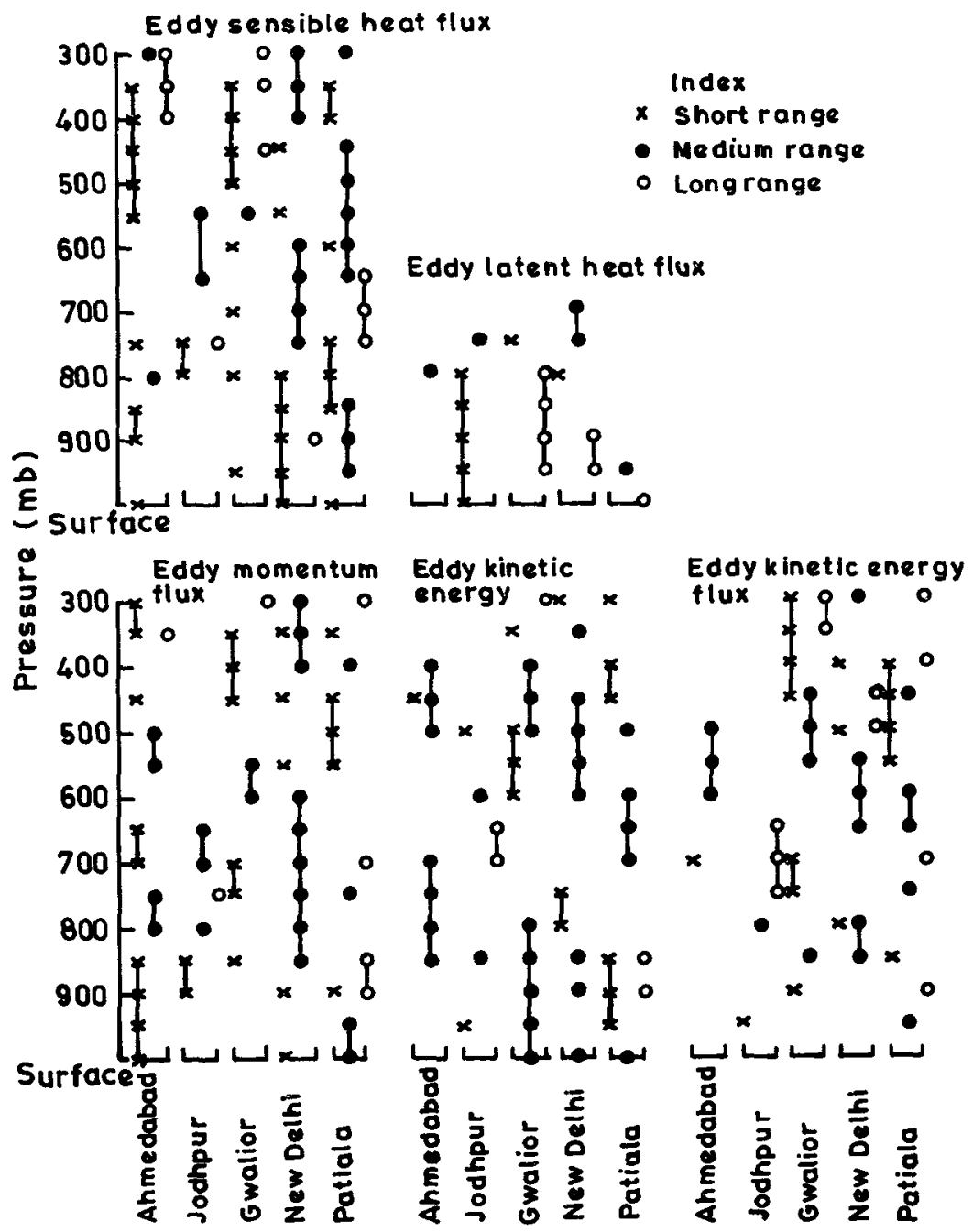

Figure 6. Periodicity in different fluxes at various stations in the vertical.

\section{Acknowledgement}

The financial support provided by UGC, New Delhi, is gratefully acknowledged.

\section{References}

Ananthakrishnan R and Keshavamurty R N 1970 On some aspects of the fluctuations in the pressure and wind fields over India during the summer and winter monsoon season; Proc. Symp. Tropical Meteorology, Honolulu, Hawaii, June 2-11, 1970, University of Hawaii, Am. Meteorol. Soc. (ed.) Paul H Kutschenreuter

Bavadekar S N and Mooley D A 1978 Computation of average precipitation over the eastern part of peninsular India during the summer monsoon from the continuity equation for atmospheric water vapour; Tellus 30 537-541 
Bhalme H N and Parasnis S S 1975 5-6 day oscillations in the pressure gradients over India during SW monsoon; Indian J. Meteorol. Hydrol. Geophys. 26 77-80

Blackman R B and Tukey J W 1958 The measurement of power spectrum (New York: Dover) p. 190

Ghosh S K, Pant M C and Dewan B N 1978 Influence of Arabian Sea on the Indian summer monsoon; Tellus 30 117-125

Gupta B R D 1972 Periodicity in the average daily strength of Siberian anticyclone from November 1967 to April 1968; Tellus 24 380-381

Gupta B R D and Singh B 1977 A power spectrum analysis of the mean daily pressure over the Mediterranean and neighbourhood during November 1967 to April 1968; Tellus 29 382-384

Madden R A and Julian P R 1972 Further evidence of global scale 5-day pressure waves; J. Atmos. Sci. 29 1464-1469

Misra B M 1972 Planetary pressure wave of 4-5 day period in the tropics; Mon. Weather Rev. 100 313-316

Murakami M 1976 Analysis of summer monsoon fluctuations over India; J. Meteorol. Soc. Jpn. 54 15-31

Rao G Appa 1981 Atmospheric energetics over India during drought and normal monsoon; Mausam 32 67-78

Rao G Appa and Ramanamurty Bh V 1972 Tropical wave disturbance over the region east of Indian Ocean; J. Meteorol. Soc. Jpn. 50 325-331

Rao G Appa and Ramanamurty Bh V 1977 Water vapour transport and vergence patterns over India during two contrasting summer monsoons; Pure Appl. Geophys. 115 491-501

Saha K R 1970 Air and water vapour transport across the equator in the western Indian Ocean during the northern summer; Tellus 22 681-687

Saha K R and Bavadekar S N 1973 Water vapour budget and precipitation over the Arabian Sea during the northern summer; Q. J. R. Meteorol. Soc. 99 273-278

Saha K R and Bavadekar S N 1977 Moisture flux across west coast of India and rainfall during southwest monsoon; Q. J. R. Meteorol. Soc. 103 370-374

Sankar Rao M 1962 On the meridional local eddy flux of heat over India; J. Atmos. Sci. $19468-473$

Sankar Rao M and Ramanadham R 1963 On the meridional local eddy flux of relative angular momentum over India; J. Atmos. Sci. 20 350-353

Singh U S and Singh R S 1987 Spectra of heat and momentum fluxes over the east coast of India during MONEX 1979; Mausam 38 411-414

Wallace J M and Chang C P 1969 Spectrum analysis of large-scale wave disturbances in the tropical lower troposphere; J. Atmos. Sci. 26 1010-1025

Yanai M, Nitta T and Hayashi Y 1968 Power spectra of large-scale disturbances over the tropical Pacific; $J$. Meteorol. Soc. Jpn. 46 308-323 